en el caso del epidídimo izquierdo muy necesaria para evitar el tratamiento de sustitución hormonal crónico.

Ninguno de los casos que hemos revisado han presentado evidencia de recidiva o diseminación, por lo que el tratamiento conservador no compromete el pronóstico de la enfermedad. Nuestro paciente está libre de enfermedad 2 años después de la cirugía, tal como lo demuestra el control ecográfico. No creemos que sea necesario un seguimiento a largo plazo para los casos unilaterales. En casos bilaterales, una valoración de la función hormonal parece apropiada.

Los leiomiomas de epidídimo son tumores poco frecuentes pero pueden presentarse en la práctica clínica diaria. El diagnóstico se basa en la palpación y el estudio ecográfico. Debido al excelente pronóstico, la exéresis podría obviarse, pero la ecografía no suele ser suficientemente fiable para el diagnóstico definitivo, por lo que la exéresis quirúrgica completa es el tratamiento más recomendable cuando nos enfrentamos a una masa sólida paratesticular. Una vez que conocemos que se trata de un leiomioma hay que evitar los tratamientos agresivos dado que, como en nuestro caso, puede aparecer otro tumor en el epidídimo contralateral, incluso años después.

\section{Agradecimientos}

Al Servicio de Anatomía Patológica y concretamente a la jefa de servicio, la Dra. Carme Admella Salvador.

B I B L I O G R A F Í A

1. Attignac P, Enarard P, Debre B. Kystes et tumeurs de l'epididyme. Encicl Med Chir (París). Rein organes genitourinaires. 1984;B10:1865.
2. Krichen Maken S, Mnif Hachicha L, Ellouze S, et al. Femizing testicular syndrome with multiple hamartomas and bilatera paratesticular leiomyomas. Rev Med Interne. 2005;26: 980-3.

3. Forte F, Scartini M, Spera E, et al. Leiomyoma of the epididymis and testicular anexa: Apropos of 3 cases. Arch Ital Urol Androl. 2004;76:119-20.

4. Mack CW, Tzeng WS, Chou CK, et al. Leiomyoma arising from the tunica albuginea of the testis: Sonographic findings. J Clin Ultrasound. 2004;32:309-11.

5. Destito A, Serrello C, Pisanti F, et al. Leiomyoma of the testis. Scand J Urol Nephrol. 1999;33:338-9.

6. Larbcharoensub BN, Sornmayura P, Ariyaprakai W. Bilateral leiomyoma of the epidydimimes. J Med Assoc Thai. 2006;89: 1051-1054.

7. Fumo MJ, Assi QA, Liroff S. Leiomyoma of the epididymis treated with partial epididimectomy. Nat Clin Pract Urol. 2006;3:504-7.

8. Cuesta Alcalá JA, Arrondo Arrondo JL, Pascual Piédrola I, et al. Diagnóstico diferencial del leiomioma de epidídimo. Aportación de un nuevo caso. Arch Esp Urol. 2001;54: 823-5.

9. Borri A, Nesi G, Bancini L, Pernice LM. Bizarre leiomyoma of the epididymis. A case report. Minerva Urol Nefrol. 2000;52: 29-31.
J. Llopis Manzanera ${ }^{a, *}$, C. Ramírez Sevilla ${ }^{a}$,
C. Ferrer Cassadób y J.A. Romero Martin ${ }^{a}$

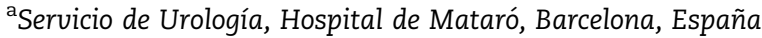

bservicio de Anatomía Patológica, Hospital de Mataró, Barcelona, España

*Autor para correspondencia.

Correo electrónico: jllopis@csdm.es (J. Llopis Manzanera).

\title{
Lactante con estenosis de uréter medio congénito tratado endourológicamente
}

\section{Endourological treatment of congenital midureteral stricture in an infant}

\section{Sr. Director:}

Presentamos el caso de un lactante derivado a nuestra unidad por diagnóstico prenatal de hidronefrosis derecha severa y riñón contralateral normal. En las exploraciones realizadas en las primeras semanas de vida se objetivó una hidronefrosis derecha severa. La cistouretrografía miccional fue normal. En el seguimiento, las ecografías mostraron un aumento progresivo de la dilatación renal derecha con dilatación de todos los grupos caliciales y adelgazamiento progresivo del parénquima renal, llegando a ser de $3 \mathrm{~mm}$. Además, se observó un uréter proximal muy dilatado y tenso (fig. 1). El renograma diurético a los 4 meses de vida mostró una disminución de la función renal diferencial derecha $(40 \%)$ con un patrón muy obstructivo. El lactante se mantuvo asintomático.

Se realizó una pielografía retrógrada con tutor ureteral de 4 Fr, encontrando una estenosis severa de uréter medio derecho con importante dilatación del uréter preestenótico. Tras lograr el paso de una guía de 0,014" hasta la pelvis renal derecha, se realizó una dilatación con balón de alta presión 


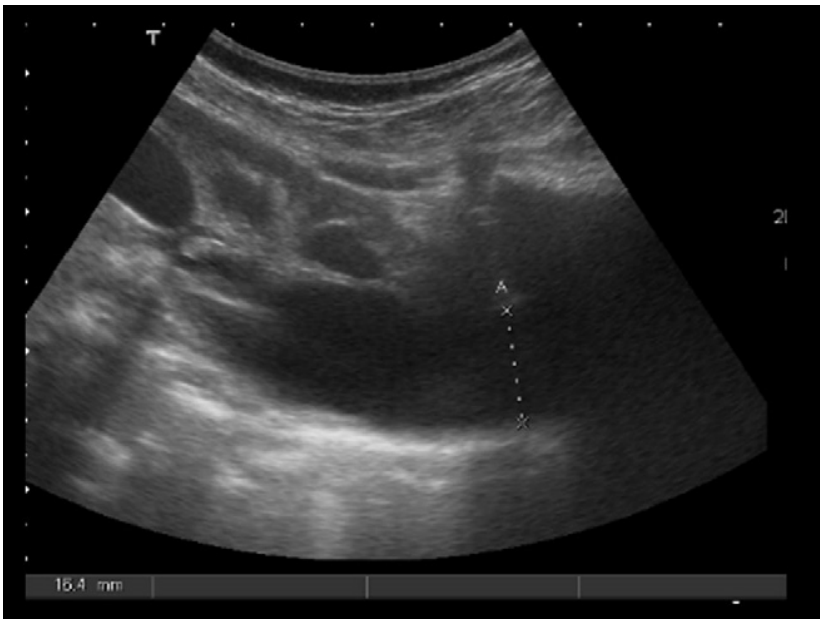

Figura 1 - Ecografía preoperatoria con dilatación del uréter proximal.

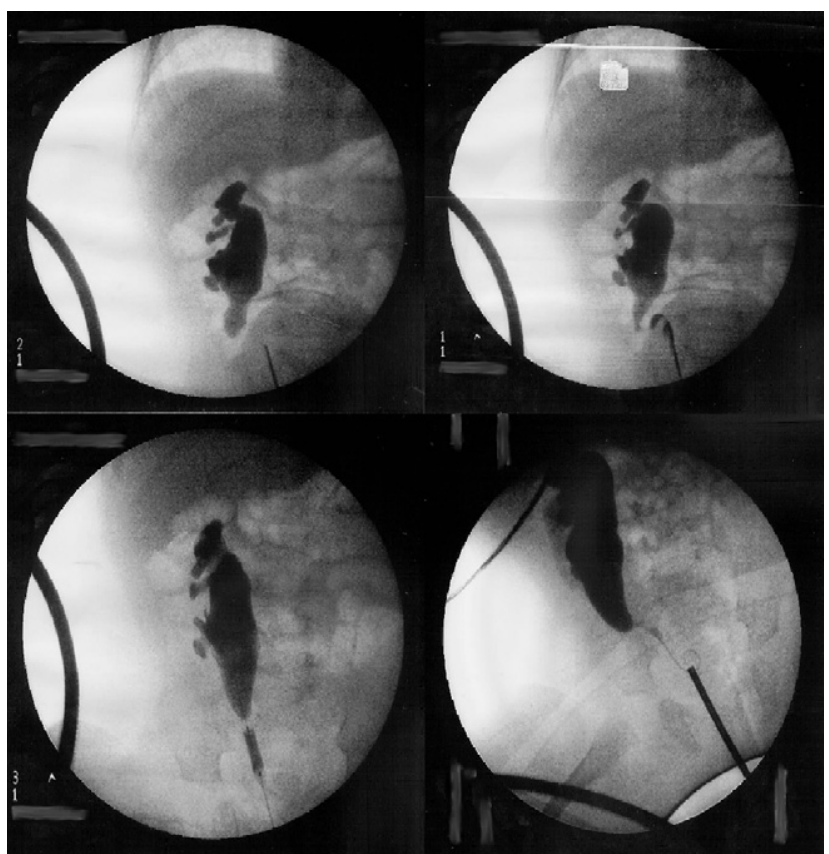

Figura 2 - Pielografía retrógrada, balón de dilatación a nivel de estenosis del uréter medio tras lograr vencer la muesca y colocación posterior de stent tipo doble J.

hasta $6 \mathrm{~mm}$ y $18 \mathrm{~atm}$ con control radioscópico. En la dilatación se observó una muesca en el inflado del balón sobre la zona estenótica que fue vencida. Tras ella se procedió a la colocación de una stent ureteral tipo doble J de 3 Fr y $12 \mathrm{~cm}$ (fig. 2). El paciente fue dado de alta hospitalaria a las $24 \mathrm{~h}$ de la intervención. El stent fue retirado a las 6 semanas de su colocación sin complicaciones, procediendo a realizar calibración del uréter sin encontrar muescas en el balón de dilatación. Este procedimiento fue realizado de forma ambulatoria. No se produjeron complicaciones intraoperatorias ni postoperatorias. En el seguimiento encontramos una normalización ecográfica desde el 4 . $^{\circ}$ mes postoperatorio. El renograma diurético al año de la intervención mostró una función renal derecha del 45\% con una normalización del patrón de la curva.

La estenosis congénita de uréter medio es una rara entidad, de difícil diagnóstico preoperatorio, que suele ser confundida con otras patologías obstructivas más frecuentes de la vía urinaria. En la actualidad encontramos menos de 30 pacientes publicados en la literatura científica en series de pequeño tamaño, siempre inferiores a 5 niños ${ }^{1}$. En una serie consecutiva de autopsias realizadas en 12.080 niños, la prevalencia de estenosis a nivel del uréter medio fue del $0,18 \%^{2}$. Pese a que los avances en el diagnóstico prenatal han aumentado la incidencia de hidronefrosis congénita, esto no ha repercutido en un aumento de la prevalencia de esta patología. Sólo la realización sistemática de pielografía retrógrada o anterógrada preoperatoria lograría definir con exactitud el punto de obstrucción y así podría realizarse un tratamiento mucho más adecuado ${ }^{1,3}$.

El tratamiento habitual para estos pacientes ha sido la ureteroureterostomía distal abierta o, en los últimos años, laparoscópica, o la ureteropielostomía en caso de encontrarse en el seno de un riñón duplicado ${ }^{1,3}$. Sin embargo, no hemos encontrando en niños ningún caso de abordaje endourológico. Este tipo de abordaje sí ha sido realizado en adultos para solucionar estenosis secundarias de uréter medio utilizando con buenos resultados la endoureterotomía anterógrada o retrógrada con láser Holmium o con Acucise ${ }^{\circledR} 6$. Presentamos un lactante con estenosis congénita de uréter medio tratado mediante dilatación retrógrada con balón, siendo probablemente el primer abordaje endourológico completo de esta entidad en niños.

Así, aun siendo una patología infrecuente, es posible que el diagnóstico esté siendo infravalorado en la actualidad debido a su complejidad diagnóstica. Prieto et $\mathrm{al}^{2}$ indican que la mayor parte de estos pacientes son erróneamente diagnosticados de megauréter primario o estenosis pieloureteral congénita. El diagnóstico de certeza debe ser realizado mediante pielografía retrógrada o anterógrada para así poder afirmar con rotundidad el punto exacto de la obstrucción ${ }^{1,3,4}$. Aunque es posible que la pielografía anterógrada aporte ventajas sobre la retrógrada al poder valorar mejor la resistencia al paso del contraste por la unión ureterovesical y disminuir la agresión sobre ella ${ }^{5}$, esta implica un abordaje percutáneo del riñón, el cual pensamos no es fácil de realizar en lactantes y aporta una morbilidad asociada no despreciable. Por ello, nosotros preferimos la pielografía retrógrada mediante el uso de tutores ureterales de bajo perfil (3 o 4 Fr).

Algunos autores han referido que, a diferencia de otras estenosis del tracto urinario, todos los pacientes de las series analizadas han requerido tratamiento quirúrgico, no siendo útil el tratamiento conservador ${ }^{1}$. Los hallazgos anatomopatológicos más frecuente son la hiperplasia asimétrica de la capa muscular del uréter con dilatación de estructuras proximales, siendo raro encontrar fibrosis de las distintas capas periuroteliales. La longitud del segmento anómalo es siempre limitada y menor de $2 \mathrm{~cm}^{2,4}$. Encontramos en todos estos hallazgos justificación para intentar realizar un tratamiento endourológico, ya que no existen compresión extrínseca del uréter ni fibrosis que vayan a implicar una alteración del crecimiento del segmento ureteral en el futuro. En nuestra única experiencia, el tiempo operatorio fue corto (20 min) al poder realizarse rápidamente tras la pielografía, 
la estancia hospitalaria fue breve al posibilitar un alta precoz a las $24 \mathrm{~h}$ de la intervención igual que nuestro equipo realiza con las dilataciones de la estenosis pieloureterales congénitas ${ }^{6}$, y la morbilidad fue mínima al no dañar la vascularización del uréter. Entre sus incovenientes encontramos la dificultad para tutorizar la vía urinaria de un lactante, aunque pensamos que es posible disponiendo del material adecuado y tras una adecuada curva de aprendizaje. La necesidad de colocar un stent tipo doble J no representa una diferencia con la técnica abierta en la que también se utiliza rutinariamente ${ }^{4}$.

B I B L I O G R A F Í A

1. Smith BG, Metwalli AR, Leach J, Cheng EY, Kropp BP. Congenital midureteral stricture in chidren diagnosed with antenatal hydronephrosis. Urology. 2004;64:1014-9.

2. Campbell MF. Clinical considerations of the anatomy, physiology, embryology, and anomalies of the urogenital tract. In: Pediatric Urology. New York: MacMillan Co.; 1937. pp. 188, 273, 287-289.
3. Prieto JC, Castellan M, Gosalbez R, Labbie A, Perez-Brayfield M. Severe congenital midureteral dilatation. J Ped Surg. 2007;42: 257-8.

4. Hwang AH, McAleer IM, Shapiro E, Miller OF, Krous HF, Kaplan GW, et al. Congenital mid ureteral strictures. J Urol. 2005;174:1999-2002.

5. Gnessin E, Yossepowitch O, Holland R, Liune PM, Lifshitz DA. Holmium laser endoureterotomy for benign ureteral stricture: A single center experience. J Urol. 2009;182:2775-9.

6. Parente A, Angulo JM, Romero R, Rivas S, Laín A, Fanjul M, et al. Resultados a medio plazo del tratamiento endourológico retrógrado con balón de la estenosis pieloureteral en niños menores de 1 año. Actas Urol Esp. 2009;33:422-8.

A. Parente Hernández*, J.M. Angulo Madero,

R.M. Romero Ruiz y S. Rivas Vila

Sección Urología Pediátrica, Servicio Cirugía Pediátrica, Hospital Infantil Gregorio Marañón, Madrid, España

*Autor para correspondencia.

Correo electrónico: parente80@hotmail.com

(A. Parente Hernández).

\section{Priapismo secundario a tratamiento por cabergolina: primera descripción de esta asociación}

\section{Priapism secondary to treatment due to cabergoline: The first description of this association}

\section{Sr. Director:}

Recientemente hemos tratado en el servicio de urgencias a un paciente de 33 años por un cuadro de priapismo debajo flujo de seis horas de evolución. Entre su antecedentes solo destacaba que padecía de un hiperprolactinoma y estaba siendo tratado con cabergolina.

El paciente nos relató que el cuadro se había producido en 2 ocasiones antes del episodio que le obligó a asistir a urgencias. El primer episodio de erección prolongada se había producido al mes de iniciar el tratamiento habiéndose autolimitado de forma espontánea en 3 horas. El segundo episodio tuvo lugar una semana antes del definitivo, cursando de la misma forma que el previo.

En nuestra asistencia se realizaron las maniobras monitorizadas de inyección de epinefrina, según los protocolos conocidos $^{1}$, sin resultar eficaz la maniobra. Definitivamente se solucionó el cuadro en quirófano, bajo anestesia raquídea, realizando una punción con lavado de cuerpos cavernosos a través del glande. El paciente actualmente ha abandonado el tratamiento y no se ha vuelto a producir el priapismo. Los niveles de prolactina, y testosterona fueron determinados al día siguiente del priapismo, resultando los mismos normales.
La cabergolina es un derivado ergótico que actúa como agonista dopaminérgico, autorizado en España para el tratamiento de los signos y síntomas asociados a la enfermedad de Parkinson $\left(\right.$ Sogilen ${ }^{\circledR}$ ) y para tratamiento de la hiperprolactinemia e inhibición de la lactancia (Dostinex ${ }^{\circledR}$ ).

La Agencia Española de Medicamentos y Productos Sanitarios emitió en noviembre de 2004 una nota informativa sobre pergolida, otro derivado ergótico también utilizado para el tratamiento de signos y síntomas de la enfermedad de Parkinson, en relación con riesgos cariovasculares similares a los encontrados para cabergolina.

Tanto para pergolida como para cabergolina, las valvulopatías observadas se producen como consecuencia de una reacción fibrótica que produce una restricción en el movimiento normal de las válvulas, generando regurgitación que en algunos casos requiere el recambio valvular. Esta reacción fibrótica se podría producir como respuesta a la afinidad que presentan estos fármacos por los receptores serotoninérgicos 5-HT2b.

En 2 estudios publicados recientemente se ha estimado la prevalencia $^{2}$ e incidencia ${ }^{3}$ de valvulopatía cardiaca asociada al tratamiento con pergolida y cabergolina, así como de otros agonistas dopaminérgicos, comparándose con la obtenida en un grupo control. 